\section{Sensitivity of Botrytis cinerea to fenpyrazamine in Japan and its disease control efficacy against the low-sensitive isolate}

\author{
Dai Hirotomi, ${ }^{1}$ Soichi Tanaka, ${ }^{2}$ \\ Fukumatsu IWAHASHI ${ }^{3}$ and Norio KIMURA ${ }^{3, *}$ \\ ${ }^{1}$ Kasai Experimental Farm, Health \& Crop Sciences Research Laboratory, \\ Sumitomo Chemical Co., Ltd., 636-2 Shionoyama, Kishiro-Cho, \\ Kasai, Hyogo, Japan \\ ${ }^{2}$ AgroSolutions, Division-International, Sumitomo Chemical Co., Ltd., \\ 2-27-1 Shinkawa, Chuo-ku, Tokyo, Japan \\ ${ }^{3}$ Health \& Crop Sciences Research Laboratory, Sumitomo Chemical \\ Co., Ltd., 4-2-1 Takatsukasa, Takarazuka, Hyogo, Japan
}

(Received May 25, 2020; Accepted August 5, 2020)

The baseline sensitivity of Botrytis cinerea to fenpyrazamine was evaluated using 323 isolates collected in Japan prior to its launch. In this study, the isolates were classified as "sensitive" and "low-sensitive" according to their mycelial growth on $10 \mathrm{mg} / \mathrm{L}$ fenpyrazamine. However, their $\mathrm{EC}_{50}$ values for the germ-tube elongations from conidia were not significantly different between these two classes. In both a pot test and a field trial, diseases caused by the sensitive and low-sensitive isolates were effectively controlled by fenpyrazamine.

Keywords: fenpyrazamine, sensitivity, low-sensitive, Botrytis cinerea.

\section{Introduction}

In the agricultural industry, gray mold (Botrytis cinerea) attacks fruits and vegetables and greatly reduces crop yield and productivity; therefore, it must be controlled. ${ }^{1)}$ Many fungicides, such as benomyl, have been developed to control gray mold. However, gray mold has a short lifecycle and produces many spores, making it a phytopathological fungus that easily acquires fungicide resistance. ${ }^{2)}$ Many of these fungi have developed resistance to commercial fungicides ${ }^{3,4)}$; thus, farmers and food-related industries await novel fungicides. Fenpyrazamine is one novel fungicide discovered and developed by Sumitomo Chemical Co., Ltd. (Tokyo, Japan). Fenpyrazamine contains an aminopyrazo-

\footnotetext{
* To whom correspondence should be addressed.

E-mail: kimuran@sc.sumitomo-chem.co.jp

Published online September 19, 2020
}

(c) BY-NC-ND (c) Pesticide Science Society of Japan 2020. This is an open access article distributed under the Creative Commons AttributionNonCommercial-NoDerivatives 4.0 International (CC BY-NC-ND 4.0) License (https://creativecommons.org/licenses/by-nc-nd/4.0/) linone structure that has never before been used in agricultural chemicals. ${ }^{5)}$ Sumitomo Chemical Co., Ltd. obtained the Japanese registration for fenpyrazamine fungicide and launched the dry flowable (DF) “Pixio ${ }^{\circledR}$ DF” in Japan in January 2014. ${ }^{5)}$

When developing a novel fungicide, the baseline sensitivities of the target fungi to the chemicals must be evaluated before its commercial introduction. Consequently, we monitored the sensitivity of $B$. cinerea isolates in Japan to fenpyrazamine and evaluated the baseline sensitivity of $B$. cinerea to fenpyrazamine.

\section{Materials and Methods}

\section{Fungal isolates}

Three hundred twenty-three $B$. cinerea isolates, collected from fields in Japan before fenpyrazamine was commercially introduced, were used in this study (Table 1 ). To isolate $B$. cinerea from decayed fruits, small pieces of decayed tissue were placed on potato dextrose agar (PDA; Nissui Pharmaceutical Co., Tokyo, Japan) containing chloramphenicol $(50 \mathrm{mg} / \mathrm{L})$ and incubated at $18^{\circ} \mathrm{C}$ for $3-5 \mathrm{~d}$. Fungal colonies similar in appearance to gray mold were transferred onto fresh PDA containing chloramphenicol. Pure cultures were then grown on PDA and incubated at $18^{\circ} \mathrm{C}$ under black/blue light to facilitate sporulation. The $\mathrm{B}$. $\mathrm{ci}$ nerea isolates were then single-spore cultured and stored at $12^{\circ} \mathrm{C}$.

\section{Chemicals}

The technical grade fenpyrazamine used for the in vitro plate assay was synthesized by Sumitomo Chemical Co., Ltd. The fenpyrazamine used for the pot test and field trial was $50 \%$ DF formulated by Sumitomo Chemical Co., Ltd. Fludioxonil (Savior SC20, Syngenta Japan, Tokyo, Japan) was purchased from an agricultural cooperative.

3. Monitoring B. cinerea sensitivity to fenpyrazamine Fenpyrazamine was dissolved in dimethyl sulfoxide (FUJIFILM Wako Pure Chemical Corporation, Osaka, Japan) to a predetermined concentration $(10 \mathrm{~g} / \mathrm{L})$ to provide the stock solution, which was added to the PDA to obtain $10 \mathrm{mg} / \mathrm{L}$ fenpyrazamine. Mycelial plugs from the colonial margins of the tested isolates precultured at $18^{\circ} \mathrm{C}$ were placed on the PDA supplemented with $10 \mathrm{mg} / \mathrm{L}$ fenpyrazamine and incubated at $18^{\circ} \mathrm{C}$ for $2 \mathrm{~d}$. The mycelial growth was assessed by visual observation. Isolates showing no mycelial growth on the PDA were defined as "sensitive isolates." Sporulation of isolates showing mycelial growth on the PDA containing fenpyrazamine was facilitated as described above. A conidial suspension $(10 \mu \mathrm{L}, \sim 50,000$ conidia $/ \mathrm{mL})$ was placed on PDA containing $2 \mathrm{mg} / \mathrm{L}$ fenpyrazamine and incubated at $18^{\circ} \mathrm{C}$ for $24 \mathrm{hr}$, and then the germ-tube elongations were assessed microscopically. The sensitivity criteria for $B$. cinerea to fenpyrazamine were as follows.

Sensitive isolates: No mycelial growth observed among the iso- 
Table 1. Collection locations of Botrytis cinerea isolates used in this study

\begin{tabular}{lcc}
\hline \multicolumn{1}{c}{ Collection locations } & Nos. of isolates & Years $^{a)}$ \\
\hline Hokkaido and Tohoku & 14 & 2008,2009 \\
Kanto & 141 & 2008,2009 \\
Kinki & 61 & 2008,2009 \\
Chugoku and Shikoku & 34 & 2008,2009 \\
Kyushu & 73 & 2008,2009 \\
\hline
\end{tabular}

a) Years in which $B$. cinerea isolates were collected

lates on the PDA containing $10 \mathrm{mg} / \mathrm{L}$ fenpyrazamine.

Low-sensitive isolates: Mycelial growth observed among the isolates on the PDA containing $10 \mathrm{mg} / \mathrm{L}$ fenpyrazamine, but the germ-tube elongations were strongly inhibited on the PDA containing $2 \mathrm{mg} / \mathrm{L}$ fenpyrazamine.

Resistant isolates: Mycelial growth observed among the isolates on the PDA containing $10 \mathrm{mg} / \mathrm{L}$ fenpyrazamine. Germtube elongations were not inhibited on the PDA containing $2 \mathrm{mg} / \mathrm{L}$ fenpyrazamine.

\section{Baseline sensitivity of $\mathrm{B}$. cinerea to fenpyrazamine}

\subsection{Sensitivity of B. cinerea mycelia}

Sixty-one randomly selected sensitive $B$. cinerea isolates were used. The mycelial sensitivity of the tested isolates was evaluated using fenpyrazamine test concentrations of $0.005-2 \mathrm{mg} / \mathrm{L}$. The $5 \mathrm{~mm}$ mycelial discs (2 discs/isolate) were placed on the PDA, and the mycelial radii were measured $2 \mathrm{~d}$ after inoculation. The half maximal effective concentration $\left(\mathrm{EC}_{50}\right)$ value of fenpyrazamine was calculated using the probit method.

The mycelial plate test of the low-sensitive isolates revealed that the dose-response curve was a rare flat slope. For example, the rates (\%) of mycelial growth inhibition due to fenpyrazamine for one low-sensitive isolate were $58.3 \%$ at $50 \mathrm{mg} / \mathrm{L}, 52.4 \%$ at $10 \mathrm{mg} / \mathrm{L}$, $57.1 \%$ at $2 \mathrm{mg} / \mathrm{L}, 59.5 \%$ at $0.4 \mathrm{mg} / \mathrm{L}$ and $51.2 \%$ at $0.08 \mathrm{mg} / \mathrm{L}$. Therefore, we determined that low-sensitive isolates were unsuitable for evaluating $\mathrm{EC}_{50}$ values with mycelial plate tests. Thus, we used only sensitive isolates for the mycelial plate tests.

4.2. Sensitivity of B. cinerea germ-tube elongation

Twenty-one sensitive and nine low-sensitive randomly selected B. cinerea isolates were used. Sensitivity of the germ-tube elongation was evaluated using fenpyrazamine test concentrations of $0.005-5 \mathrm{mg} / \mathrm{L}$. The length of each germ tube ( 25 germ tubes/ isolate) was measured microscopically. The $\mathrm{EC}_{50}$ value of fenpyrazamine was calculated using the probit method.

5. Efficacy of fenpyrazamine against a low-sensitive B. cinerea isolate in a pot test

One sensitive and one low-sensitive $B$. cinerea isolate were used. The $50 \%$ DF of fenpyrazamine was diluted in deionized water to a predetermined concentration $(250 \mathrm{mg} / \mathrm{L}$ and $62.5 \mathrm{mg} / \mathrm{L})$. The solution was sprayed onto cucumber cotyledons (four cucumbers/treatment) until run-off occurred. After drying, $10 \mu \mathrm{L}$ of PDA gel containing conidia from the test isolate $(\sim 50,000$ conidia/mL) was inoculated on cotyledon leaves and incubated at $12^{\circ} \mathrm{C}$ and high humidity (100\%) in the dark for $5 \mathrm{~d}$, and then the lesional radii were measured. Differences in the lesional radii between the fenpyrazamine-treated and untreated plots were determined using the Tukey-Kramer method $(p<0.05)$.

6. Efficacy of fenpyrazamine against a low-sensitive B. cinerea isolate in a field trial

The field trial was conducted at the Kasai Experimental Farm of Sumitomo Chemical Co., Ltd. in Hyogo Prefecture. Eggplants were used for the trial. The plot size was five plants/plot (including one blank plant), with three replicates per treatment. One sensitive and one low-sensitive isolate were used as inocula. Diseased eggplant fruits were placed on each blank plant. Two fruits/plant were placed after the first fungicide application. Formulated fungicides were diluted in deionized water to registered concentrations. Once weekly, for a total of five applications, fungicides were sprayed until run-off occurred. Diseased and healthy fruits were counted at each assessment just before the fungicide application, and diseased fruits were removed. The final assessment was conducted $7 \mathrm{~d}$ after the final fenpyrazamine application. The percentage of diseased fruits was calculated as follows:

$$
\begin{aligned}
& \% \text { diseased fruits } \\
& \begin{aligned}
=100 \times[ & 1-(\text { total number of diseased fruits)/ } \\
& \text { (total number of healthy fruits } \\
& + \text { total number of diseased fruits })]
\end{aligned}
\end{aligned}
$$

Differences in gray mold incidence between fungicide-treated and untreated plots were determined using the Tukey-Kramer method $(p<0.05)$.

\section{Results and Discussion}

\section{Monitoring B. cinerea sensitivity to fenpyrazamine}

Among the 323 isolates, 268 were sensitive to fenpyrazamine $(>80 \%$ ), and 55 were low-sensitive (Table 2). No resistant isolates were detected. The high percentage of sensitive isolates indicated that most B. cinerea in Japan were sensitive or lowsensitive to fenpyrazamine before its commercial introduction. Fenpyrazamine acts by inhibiting 3-keto reductase in the ergosterol biosynthetic pathway. ${ }^{5}$ Four 3-keto reductase inhibitorresistant phenotypes HydR1, HydR2, HydR3 ${ }^{-}$, and $\mathrm{HydR}^{+}$have been reported in Botrytis field isolates. ${ }^{6}$ The sensitivity of the low-sensitive isolates in this study suggests that they may have

Table 2. Sensitivity of Botrytis cinerea isolates to fenpyrazamine

\begin{tabular}{ccc}
\hline Sensitive $^{a)}$ & Low-sensitive $^{b)}$ & Resistant $^{c)}$ \\
\hline $268(83 \%)$ & $55(17 \%)$ & 0 \\
\hline
\end{tabular}

${ }^{a}$ No mycelial growth observed among the isolates on the PDA containing $10 \mathrm{mg} / \mathrm{L}$ fenpyrazamine. ${ }^{b}$ Mycelial growth observed among the isolates on the PDA containing $10 \mathrm{mg} / \mathrm{L}$ fenpyrazamine, but the germtube elongations were strongly inhibited on the PDA containing $2 \mathrm{mg} / \mathrm{L}$ fenpyrazamine. ${ }^{c)}$ Mycelial growth observed among the isolates on the PDA containing $10 \mathrm{mg} / \mathrm{L}$ fenpyrazamine. Germ-tube elongations were not inhibited on the PDA containing $2 \mathrm{mg} / \mathrm{L}$ fenpyrazamine. 
been HydR1 or HydR2. Leroux et al. $(2002)^{6)}$ described HydR1 as being grouped within Botrytis pseudocinerea, while HydR2 was $B$. cinerea. In accordance with a previous report, ${ }^{7)}$ we determined whether the low-sensitive isolates were $B$. cinerea. Twenty-three low-sensitive randomly selected isolates were used, and all 23 were identified as B. cinerea (data not shown); thus, the low-sensitive isolates from Japan were HydR2-type-resistant $B$. cinerea. Albertini and Leroux $(2004)^{8)}$ reported that HydR2 has no mutation in the 3-keto reductase gene. Therefore, we amplified and sequenced a 3-keto reductase gene (erg27) from 15 randomly selected low-sensitive isolates. Among them, 12 had both P57A and A378T mutations, while three had no mutations. However, no distinguishable differences were found in their sensitivities to fenpyrazamine (data not shown). Additionally, the erg27 genes of 19 randomly selected sensitive isolates were amplified and sequenced. Only one sensitive isolate had both P57A and A378T mutations (data not shown). Although Yin et al. reported that these two mutations lead to partial resistance to fenhexamid, which targets 3-keto reductase, ${ }^{9)}$ these two mutations were also detected in one sensitive isolate $\left(\mathrm{EC}_{50}: 0.013 \mathrm{mg} / \mathrm{L}\right.$; data not shown). Thus, we hypothesize that the two mutations do not affect the antifungal activity of fenpyrazamine against $B$. cinerea. Further studies are needed to determine why the results differed between the previous report ${ }^{9)}$ and the current study.

\section{Baseline sensitivity of $\mathrm{B}$. cinerea to fenpyrazamine}

For mycelial sensitivity, the $\mathrm{EC}_{50}$ value showed a single peak centered at $0.0125-0.025 \mathrm{mg} / \mathrm{L}$ (Fig. 1). The average $\mathrm{EC}_{50}$ was $0.020 \pm 0.0097 \mathrm{mg} / \mathrm{L}$. Sixty-one sensitive isolates had no mycelial growth on PDA containing $0.5 \mathrm{mg} / \mathrm{L}$ fenpyrazamine. Conversely, mycelia from the low-sensitive isolates grew on PDA containing $10 \mathrm{mg} / \mathrm{L}$ fenpyrazamine. B. cinerea has two types of mycelial sensitivity to fenpyrazamine: sensitive and low-sensitive. For germ-tube-elongation sensitivity, the $\mathrm{EC}_{50}$ value of the sensitive and low-sensitive isolates showed a single peak centered at $0.02-0.1 \mathrm{mg} / \mathrm{L}$. The average $\mathrm{EC}_{50}$ of the sensitive isolates was

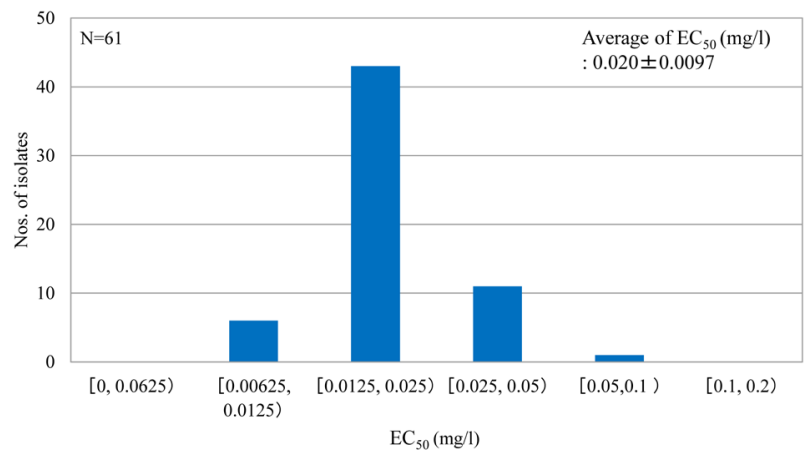

Fig. 1. Baseline mycelial sensitivity of Botrytis cinerea isolates in Japan. Sixty-one randomly selected sensitive isolates were used. Stock fenpyrazamine solution was added to PDA to form test concentrations of $0.005-2 \mathrm{mg} / \mathrm{L}$. The $5 \mathrm{~mm}$ disks of marginal mycelia of tested isolates precultured at $18^{\circ} \mathrm{C}$ were placed on the PDA, and the mycelial radii were measured $2 \mathrm{~d}$ after inoculation. The $\mathrm{EC}_{50}$ value was calculated using the probit method. $[\mathrm{a}, \mathrm{b})$ means $\mathrm{a} \leq \mathrm{EC}_{50}<\mathrm{b}$.

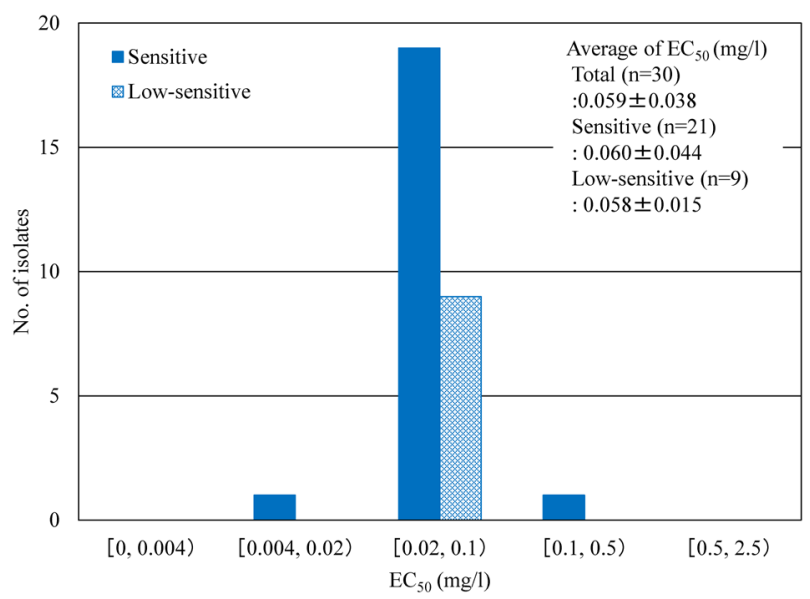

Fig. 2. Baseline conidial sensitivity of Botrytis cinerea isolates in Japan. Twenty-one sensitive and nine low-sensitive randomly selected isolates were used. Stock fenpyrazamine solution was added to PDA to form test concentrations of $0.005-5 \mathrm{mg} / \mathrm{L}$. The conidial suspension $(10 \mu \mathrm{L}, \sim 50,000$ conidia/mL) was placed on the PDA and incubated at $18^{\circ} \mathrm{C}$ for $24 \mathrm{hr}$, and then the germ tube lengths ( 25 germ tubes/isolate) were measured microscopically. The $\mathrm{EC}_{50}$ value was calculated using the probit method. [a, b) means $\mathrm{a} \leq \mathrm{EC}_{50}<\mathrm{b}$.

$0.060 \pm 0.044 \mathrm{mg} / \mathrm{L}$, which did not significantly differ from the average $\mathrm{EC}_{50}$ of the low-sensitive isolates $(0.058 \pm 0.015 \mathrm{mg} / \mathrm{L}$; Fig. 2). The average $\mathrm{EC}_{50}$ of the 30 sensitive and low-sensitive isolates together was $0.059 \pm 0.038$. These $\mathrm{EC}_{50}$ values of $0.020 \mathrm{mg} / \mathrm{L}$ and $0.059 \mathrm{mg} / \mathrm{L}$ represent the baseline sensitivity levels of $B$. cinerea in Japan before introducing fenpyrazamine.

\section{Efficacy of fenpyrazamine against a low-sensitive isolate in a pot test}

The mean radius of the low-sensitive isolate-induced lesions in the untreated pot was $19 \mathrm{~mm}$, which was comparable to the radii of the lesions induced by a sensitive isolate (mean, $20 \mathrm{~mm}$ ). This indicated that the pathogenicity of the low-sensitive isolate was nearly equal to that of the sensitive isolate. Fenpyrazamine showed high efficacy against the low-sensitive isolate, even at $62.5 \mathrm{mg} / \mathrm{L}$ (Fig. 3), which is $25 \%$ of the registered concentration. The efficacy against the low-sensitive isolate was comparable to that against the sensitive isolate (Fig. 3), which indicated that the low-sensitive isolate did not affect fenpyrazamine efficacy in the pot test.

\section{Efficacy of fenpyrazamine against a low-sensitive isolate in a field trial}

Of the fruits, $21.4 \%$ were diseased. The disease pressures were moderate to strong enough to evaluate the fungicidal efficacy. Monitoring the untreated pots revealed that the frequency of the low-sensitive isolate was approximately $32 \%$, which was sufficient to evaluate the fungicidal efficacy against low-sensitive isolate. Under these conditions, fenpyrazamine showed a high efficacy against gray mold in the presence of low-sensitive isolate (2.5\% of fruits were diseased). The efficacy was comparable to that of the reference chemical fludioxonil $(2.8 \%$ of fruits were diseased), indicating that a low-sensitive isolate did not affect 


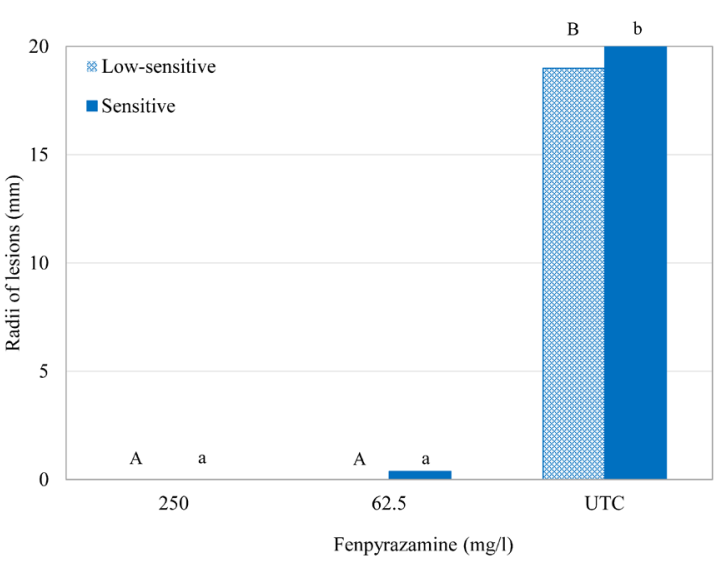

Fig. 3. Comparison of fenpyrazamine efficacy against low-sensitive and sensitive Botrytis cinerea isolates. The $50 \%$ dry flowable fenpyrazamine was diluted in deionized water to a predetermined concentration. The solution was sprayed onto cucumber cotyledons (four cucumbers/treatment) until run-off occurred. After drying, $10 \mu \mathrm{L}$ of PDA gel containing conidia of a tested isolate $(\sim 50,000$ conidia/mL $)$ was inoculated onto cotyledon leaves and incubated at $12^{\circ} \mathrm{C}$ and high humidity (100\%) in the dark for $5 \mathrm{~d}$. The lesional radii were then measured. UTC means untreated control. Different letters indicate statistical differences as per the Tukey-Kramer method $(p<0.05)$.

the fenpyrazamine efficacy even in a field trial (Table 3). This is the first report to show the high efficacy of fenpyrazamine, which targets 3-keto reductase, against a low-sensitive B. cinerea isolate at a practical field level.

In this study, we evaluated the sensitivity levels of $>300$ isolates collected in Japan before the commercial introduction of fenpyrazamine. Although we detected low-sensitive isolates at a low frequency and detected no resistant isolates, we determined that low-sensitive isolates do not affect fenpyrazamine efficacy.

$B$. cinerea sporulates profusely, and dry conidia are dispersed through the air to susceptible crops. ${ }^{1)}$ Thus, $B$. cinerea inocula are conidia, not mycelia. Although the mycelia of low-sensitive isolates exhibited low sensitivity to fenpyrazamine, conidia of the low-sensitive isolates were equally as sensitive as the sensitive isolates. Consequently, fenpyrazamine showed good disease-control performance in the presence of low-sensitive isolates in the field trial. We hypothesize that very few $B$. cinerea isolates in Japan were resistant to fenpyrazamine before its commercial release. At present, fenpyrazamine resistance remains unreported in Japan. However, according to Fungicide Resistance Action Committee classifications, 3-keto reductase inhibi- tors are classified as low-medium risk, ${ }^{10)}$ and $B$. cinerea is classified as the causal agent of high-risk disease. ${ }^{2)}$ Isolates resistant to 3-keto reductase inhibitors have been reported in the European Union ${ }^{10-13)}$ and the United States. ${ }^{14-18)}$ We believe that the situation in Japan is likely similar. Consequently, we should continue to survey for resistant isolates in the field. If resistant isolates are identified, then the sensitivity of $B$. cinerea isolates to fenpyrazamine should be monitored throughout Japan.

\section{References}

1) G. Holz, S. Coertze and B. Williamson: "Botrytis: Biology, Pathology and Control," eds. by Y. Elad, B. Williamson, P. Tudzynski and N. Delen, Springer, Dordrecht, pp. 9-24, 2007.

2) FRAC: (Fungicide Resistance Action Committee) Pathogen risk list: https://www.frac.info/docs/default-source/publications/pathogenrisk/frac-pathogen-list-2019.pdf, 2019. (Accessed 14 May, 2020)

3) J. H. Jiang, L. S. Ding, T. J. Michailides, H. Y. Li and Z. H. Ma: Pestic. Biochem. Physiol. 93, 72-76 (2009).

4) C. K. Myresiotis, G. S. Karaoglanidis and K. Tzavella-Klonari: Plant Dis. 91, 407-413 (2007).

5) N. Kimura and F. Iwahashi: J. Pestic. Sci. 40, 2-6 (2014).

6) P. Leroux, D. Debieu, C. Albertini, A. Arnold, J. Bach, F. Chapeland, E. Fournier, R. Fritz, M. Gredt, M. Hugon, C. Lanen, C. Malosse and G. Thebaud: "Modern Fungicides and Antifungal Compounds III," eds. by H. W. Dehne, U. Gisi, H. K. Kuck, P. E. Russel and H. Lyr, AgroConcept, Bonn, pp. 29-40 (2002).

7) E. Fournier, C. Levis, D. Fortini, P. Leroux, T. Giraud and Y. Brygoo: Mycologia 95, 251-261 (2003).

8) C. Albertini and P. Leroux: Eur. J. Plant Pathol. 110, 723-733 (2004).

9) D. Yin, S. Wu, N. Liu, Y. Yina and Z. Maa: Pest Manag. Sci. 72, 15401548 (2016).

10) FRAC: (Fungicide Resistance Action Committee) Code list: https:// www.frac.info/docs/default-source/publications/frac-code-list/fraccode-list-2020-final.pdf?sfvrsn $=8301499 a \_2,2020$. (Accessed 14 May, 2020)

11) P. Leroux, R. Fritz, D. Debieu, C. Albertini, C. Lanen, J. Bach, M. Gredt and F. Chapeland: Pest Manag. Sci. 58, 876-888 (2002).

12) S. Fillinger, P. Leroux, C. Auclair, C. Barreau, C. A. Hajj and D. Debieu: Antimicrob. Agents Chemother. 52, 3933-3940 (2008).

13) R. W. S. Weber: Plant Dis. 95, 1263-1269 (2011).

14) A. Achour and N. A. Peres: Plant Dis. 98, 1131-1137 (2014).

15) A. Grabke, D. Fernández-Ortuño and G. Schnabel: Plant Dis. 97, 271-276 (2013).

16) A. Amiri, S. M. Heath and N. A. Peres: Plant Dis. 97, 393-401 (2013).

17) G. W. Moorman, A. S. Walker and S. May: Plant Dis. 96, 147 (2012).

18) S. Saito, L. C. Davidson and W. F. Wilcox: Phytopathology 101, S158 (2011).

Table 3. Efficacy of fenpyrazamine against gray mold on eggplant in a field trial in Hyogo Prefecture in the presence of low-sensitive Botrytis cinerea isolate $^{a)}$

\begin{tabular}{lcccccr}
\hline \multirow{2}{*}{ Chemical } & \multirow{2}{*}{ Conc. $\mathrm{mg} / \mathrm{L}$} & \multicolumn{3}{c}{ \% of diseased fruits } \\
\cline { 4 - 7 } & & & 1st rep. & 2nd rep. & 3rd rep. & Average \\
\hline Fenpyrazamine & $50 \mathrm{DF}$ & 250 & $3.5(3 / 86)^{c)}$ & $1.2(1 / 82)$ & $2.7(2 / 75)$ & $2.5 \mathrm{a}^{b)}$ \\
Fludioxonil & 20SC & 200 & $0.0(0 / 65)$ & $5.3(6 / 114)$ & $3.2(2 / 63)$ & $2.8 \mathrm{a}$ \\
Untreated & & & $33.0(32 / 97)$ & $14.6(13 / 89)$ & $16.7(8 / 40)$ & $21.4 \mathrm{~b}$ \\
\hline
\end{tabular}

\footnotetext{
${ }^{a)}$ Frequency of low-sensitive isolate: $32 \%{ }^{b}$ Different lowercase letters indicate statistical differences as assessed by the Tukey-Kramer method $(p<0.05)$.
} ${ }^{c}$ Total number of diseased fruits/total number of diseased and healthy fruits. 